\title{
How should I wean my next intra-aortic balloon pump? Differences between progressive volume weaning and rate weaning
}

\author{
Francesco Onorati, MD, PhD, Francesco Santini, MD, Enrico Amoncelli, MD, Francesco Campanella, MD, \\ Bartolomeo Chiominto, MD, Giuseppe Faggian, MD, and Alessandro Mazzucco, MD
}

Objective: Although the intra-aortic balloon pump is the most used ventricular assist device, no study has ever evaluated the best weaning method. We compared 2 different intra-aortic balloon pump weaning methods.

\begin{abstract}
Methods: Thirty consecutive patients needing an intra-aortic balloon pump because of perioperative low-output cardiac syndrome were randomized to be weaned by ratio (4 consecutive hours of a 1:2 assisting ratio followed by 1 hour of a 1:3 ratio; group R) or by progressive volume deflation (10\% of total volume every hour for 5 consecutive hours; 15 patients, group V). A duration of 5 hours was set a priori as the weaning duration. The weaning protocol was started when the cardiac index was greater than $2.5 \mathrm{~L} / \mathrm{min} / \mathrm{m}^{2}$, the central venous pressure was $12 \mathrm{~mm} \mathrm{Hg}$ or less, the blood lactate was less than $2.5 \mathrm{mmol} / \mathrm{L}$, the mean arterial pressure was greater than 65 $\mathrm{mm} \mathrm{Hg}$, and the preserved urine output $(\geq 1 \mathrm{~mL} / \mathrm{kg} / \mathrm{hr}$ ) lasted for at least 5 consecutive hours before weaning. The cardiac index, indexed systemic vascular resistance, cardiac cycle efficiency, and central venous pressure were registered at 9 points (T0, start; T1 to T5, the first 5 weaning hours; T6, 2 hours after withdrawal; T7, 12 hours after withdrawal; and T8, at intensive care unit discharge) using the pressure recording analytical method. The interval from intra-aortic balloon pump withdrawal to intensive care unit discharge, weaning failure, perioperative troponin I, and lactate (same points) were compared.
\end{abstract}

Results: All patients, except for 1 belonging to group $\mathrm{R}(P=1.0)$, were successfully weaned. Group $\mathrm{V}$ had better preserved cardiac index, indexed systemic vascular resistance, cardiac cycle efficiency, and central venous pressure (group*time $P=.0001)$. Group R had worse cardiac index from T5 to T8 $(P \leq .0001)$, indexed systemic vascular resistance from T2 to T8 $(P \leq .004)$, cardiac cycle efficiency from T3 to T8 $(P \leq .001)$, central venous pressure from T4 to T8 $(P \leq .0001)$, and a longer interval from intra-aortic balloon pump withdrawal to intensive care unit discharge $(P=.0001)$. The lactate level was lower in group $\mathrm{V}$ from $\mathrm{T} 5$ to $\mathrm{T} 8(P \leq .027$; group*time $P=.001)$.

Conclusions: Intra-aortic balloon pump weaning by volume deflation allowed better hemodynamic and metabolic parameters. (J Thorac Cardiovasc Surg 2013;145:1214-21)

Earn CME credits at

http://cme.ctsnetjournals.org

Since the demonstration in previous decades of the efficacy of intra-aortic balloon counterpulsation (IABP) in different conditions of cardiac failure, ${ }^{1-3}$ its use has widened to include hemodynamic support in high-risk procedures, refractory unstable angina, acute myocardial infarction and its complications, low cardiac output syndrome (LCOS), difficulties in weaning from cardiopulmonary bypass, intractable ventricular arrhythmias, and so forth. ${ }^{3,4}$ Because

From the Division of Cardiac Surgery, University of Verona Medical School, Verona, Italy.

Disclosures: Authors have nothing to disclose with regard to commercial support. Received for publication Jan 9, 2012; revisions received March 14, 2012; accepted for publication March 22, 2012; available ahead of print April 23, 2012.

Address for reprints: Francesco Onorati, MD, PhD, Division of Cardiac Surgery, University of Verona Medical School, Piazzale Stefani 1, Verona 37126, Italy (E-mail: frankono@libero.it).

0022-5223/\$36.00

Copyright (c) 2013 by The American Association for Thoracic Surgery doi:10.1016/j.jtcvs.2012.03.063 of the progressive referral of sicker patients for interventional and surgical procedures, IABP indications have recently widened further to consider include this therapy as a prophylactic tool in high-risk procedures. ${ }^{5}$ Although the exact prevalence of IABP is still far from assessed, a recent survey estimated its use in 52,000 patients per year in the United States. ${ }^{6}$ In 2001, the Benchmark Registry reported the results of IABP use on more than 16,000 patients, with LCOS the most prevalent indication. ${ }^{3}$

Although IABP currently represents the first-line left ventricular assist device in patients with LCOS, no study has assessed which is the best method to wean a patient from the IABP of the 2 available methods: volume deflation or rate reduction. Therefore, it was the aim of the present pilot study to investigate, for the first time in humans, the hemodynamic response and leakage of biochemical markers of myocardial and peripheral ischemia (troponin I and lactate) using these 2 weaning methods for IABP discontinuation.

\section{METHODS \\ Patients \\ From January 2009 to October 2011, of 4390 patients undergoing car- diac surgery with cardiopulmonary bypass at our institution, 33}



Abbreviations and Acronyms
AMI = acute myocardial infarction
$\mathrm{CCE}=$ cardiac cycle efficiency
$\mathrm{CI}=$ cardiac index
$\mathrm{CVP}=$ central venous pressure
IABP $=$ intra-aortic balloon pump
ICU = intensive care unit
ISVR $=$ indexed systemic vascular resistance
LCOS $=$ low cardiac output syndrome

consecutive patients $(0.75 \%)$ requiring an intra- or postoperative IABP because of LCOS refractory to inotropic or volume support were preliminary scheduled for enrollment in the present pilot study. Three patients who also required extracorporeal circulation and membrane oxygenation for progressive heart failure were eventually excluded. Thus, 30 patients able to start a weaning trial were finally enrolled.

LCOS was diagnosed in the presence of an inadequate cardiac index (CI, $\leq 2.2 \mathrm{~L} / \mathrm{min} / \mathrm{m}^{2}$ for $>30$ minutes); reduced urine output $(<1 \mathrm{~mL} / \mathrm{kg} /$ $\mathrm{hr}$ ); metabolic acidosis (elevated serum lactate $[>4 \mathrm{mmol} / \mathrm{L}]$ ) despite inotropic support and adequate correction of preload, afterload, electrolytes, blood gas abnormalities, and glycemic control; and without evidence of cardiac tamponade. ${ }^{7}$

The IABP catheter was always inserted percutaneously, always using the sheathless technique ( $8 \mathrm{~F}, 34$ or $40 \mathrm{~mL}$ according to the patient's body surface area; Datascope, Fairfield, NJ) and connected to a Datascope pump (Datascope). The correct positioning was always assessed by transesophageal echocardiography and confirmed by chest radiography. The preoperative/intraoperative data, timing, and indications for IABP are reported in Table 1.

\section{Weaning Trial}

The duration of the weaning trial was established a priori to last for 5 consecutive hours, according to our traditional institutional policy. The weaning trial was started when the CI was greater than $2.5 \mathrm{~L} / \mathrm{min} / \mathrm{m}^{2}$, the central venous pressure (CVP) was $12 \mathrm{~mm} \mathrm{Hg}$ or less, the blood lactate level was less than $2.5 \mathrm{mmol} / \mathrm{L}$, the mean arterial pressure was greater than $65 \mathrm{~mm} \mathrm{Hg}$, and the preserved urine output ( $\geq 1 \mathrm{~mL} / \mathrm{kg} / \mathrm{hr}$ ) lasted for at least 5 consecutive hours. Patients were randomized by lottery to the intensive care unit (ICU) with 2 different weaning strategies. Fifteen patients (group $\mathrm{R}$ ) were randomized to be weaned using the rate-reduction weaning protocol, consisting of switching IABP from a 1:1 to a 1:2 electrocardiographiccoupled assisting ratio for the first 4 consecutive hours and from a 1:2 to a 1:3 ratio for the last (fifth) hour of the weaning trial, followed by IABP withdrawal. The other 15 patients (group V) were allocated to the volume-deflation weaning protocol, consisting of progressive volume deflation $(10 \%$ of the full volume of inflation every hour for 5 consecutive hours to reach $50 \%$ of the total volume of inflation at the end of the trial), followed by IABP withdrawal.

The weaning trial was stopped and IABP therapy prolonged beyond the fifth hour in the case of acute hemodynamic instability with signs of renewed LCOS. These patients were immediately switched to full IABP assistance (1:1 ratio with full-volume inflation) and considered to have weaning failure and were withdrawn from the trial.

\section{Hemodynamic Monitoring and Collected Data}

The pressure recording analytical method was used for hemodynamic monitoring and consisted of a beat-by-beat evaluation of the CI, indexed systemic vascular resistance (ISVR), and cardiac cycle efficiency (CCE), derived from the arterial-pressure waveform. ${ }^{8,9}$ The degree of CI changes during the weaning trial was considered as the primary endpoint of the study (see the section "Statistical Analysis"). The pressure recording analytical method is based on the mathematical analysis of the arterial pressure profile changes and has been recently validated for patients with LCOS and unstable patients undergoing IABP and/or receiving highdose inotropic support after cardiac surgery. ${ }^{8}$ It has been also recognized as the only less-invasive method able to correlate with the thermodilution calculations obtained using the Swan-Ganz catheter during IABP and to compare with the hemodynamic parameters calculated by direct oxygen Fick method or with transesophageal echocardiography in different hemodynamic conditions. ${ }^{8}$ From the continuous recording of the arterialpressure waveform, the algorithm of the machine computes the CCE, which provides information about left ventricular wall stress and the heart's effort to maintain adequate blood flow and oxygen delivery to the tissues. ${ }^{9}$ $\mathrm{CCE}$ is a nondimensional number ranging from +1 to negative values. Positive CCE values represent a better coupling between cardiac function and energy expenditure (ie, lower left ventricular wall stress). Negative $\mathrm{CCE}$ values represent greater energy expenditure and left ventricular wall stress. ${ }^{9}$ Hemodynamic indexes were collected using the pressure recording analytical method at 9 different points (T0, start of the weaning trial; T1 to T5, the first 5 weaning hours; T6, 2 hours after withdrawal; T7, 12 hours after withdrawal; and T8, ICU discharge). The CVP was recorded at the same points and expressed as millimeters of mercury.

Serum troponin I (monoclonal antibodies; Siemens Medical Solutions Diagnostics, Terrytown, NY) and lactate (RapidLab1265 AutomaticQC Cartridge; Siemens Healthcare Italia, Milan, Italy) were measured at the same 9 points, just as for the hemodynamic indexes. Venous blood for the detection of troponin I and lactate was always sampled from the distal line of the central venous catheter. ${ }^{7}$ The changes in the other hemodynamic variables and modifications of troponin I and lactate values during the weaning trial were considered the secondary endpoints of the study.

The prospectively recorded data also included the interval from IABP withdrawal to ICU discharge, inotropic support or the need for increment inotropic support after withdrawal, LCOS after withdrawal, the need for reinstitution of IABP after withdrawal, acute myocardial infarction (AMI) after withdrawal, paroxysmal/persistent atrial fibrillation after withdrawal, ICU stay, hospital stay, and hospital mortality. Inotropic support was categorized as none, low (dopamine $\leq 5 \mu \mathrm{g} / \mathrm{kg} / \mathrm{min}$ ), medium (dopamine $>5$ $\mu \mathrm{g} / \mathrm{kg} / \mathrm{min}$ and $\leq 10 \mu \mathrm{g} / \mathrm{kg} / \mathrm{min}$ or dobutamine $<5 \mu \mathrm{g} / \mathrm{kg} / \mathrm{min}$ ), and high (dopamine $>10 \mu \mathrm{g} / \mathrm{kg} / \mathrm{min}$ with or without dobutamine $>5 \mu \mathrm{g} / \mathrm{kg} / \mathrm{min}$ with or without epinephrine at any dose), according to institutional policy. ${ }^{7}$ Postwithdrawal LCOS was defined as a CI of $\leq 2.2 \mathrm{~L} / \mathrm{min} / \mathrm{m}^{2}$ for more than 30 minutes with reduced urine output $(<1 \mathrm{~mL} / \mathrm{kg} / \mathrm{hr})$, metabolic acidosis with elevated serum lactate $(>4 \mathrm{mmol} / \mathrm{L})$, despite adequate glycemic control, without any evidence of cardiac tamponade and despite inotropic support and adequate correction of preload, afterload, electrolyte, and blood gas abnormalities. ${ }^{7}$ Postwithdrawal inotropic support was increased after IABP discontinuation in the presence of signs of LCOS and titrated on the hemodynamic indexes. Postwithdrawal LCOS refractory to inotropic support mandated reinstitution of IABP therapy and was also considered a weaning failure. Postwithdrawal AMI was defined according to the European Society of Cardiology/American College of Cardiology Foundation/ American Heart Association/World Heart Federation expert consensus document on the definition of AMI. ${ }^{10}$ Paroxysmal or persistent atrial fibrillation was defined according to the Heart Rhythm Society/European Heart Rhythm Association/European Cardiac Arrhythmia Society Expert Consensus Statement, as already reported. ${ }^{11}$ The ICU stay was the days spent in the ICU. A eupnoeic, extubated patient, with the absence of IABP support and with stable hemodynamic status (as confirmed by a CI $>2.5$ $\mathrm{L} / \mathrm{min} / \mathrm{m}^{2}$, a CVP of $\leq 12 \mathrm{~mm} \mathrm{Hg}$, a blood lactate level $<2.5 \mathrm{mmol} / \mathrm{L}$, a mean arterial pressure $>65 \mathrm{~mm} \mathrm{Hg}$, and preserved diuresis of $\geq 1 \mathrm{~mL} /$ $\mathrm{kg} / \mathrm{hr}$ ) fulfilled the indication for an ICU discharge. The hospital stay was the days spent in the hospital after discharge from the ICU. 
TABLE 1. Preoperative and intraoperative data and timing and indications for IABP

\begin{tabular}{lccc}
\hline \multicolumn{1}{c}{ Variable } & Group V & Group R & P value \\
\hline Age (y) & $73.2 \pm 5.0$ & $74.1 \pm 4.3$ & .616 \\
Male gender & $11(73.3)$ & $9(60.0)$ & .439 \\
Diabetes mellitus & $12(80.0)$ & $11(73.3)$ & .666 \\
Hypertension & $10(66.7)$ & $10(66.7)$ & 1.0 \\
Dyslipidemia & $10(66.7)$ & $8(53.3)$ & .456 \\
Smoking & $11(73.3)$ & $10(66.7)$ & .690 \\
COPD & $13(86.7)$ & $12(80.0)$ & .624 \\
Kidney disease & $11(73.3)$ & $10(66.7)$ & .690 \\
Logistic euroSCORE & $14.1 \pm 2.8$ & $13.4 \pm 2.9$ & .498 \\
Preoperative LVEF (\%) & $37.7 \pm 3.0$ & $38.2 \pm 4.3$ & .736 \\
ACC time (min) & $94.1 \pm 15.7$ & $86.8 \pm 15.3$ & .211 \\
CPB time (min) & $154.6 \pm 38.2$ & $130.0 \pm 36.5$ & .082 \\
CABG (n) & $3.2 \pm 0.7$ & $2.8 \pm 0.5$ & .140 \\
Concomitant procedure & $13(86.7)$ & $12(80.0)$ & .624 \\
$\quad$ Mitral valve surgery & $8(53.3)$ & $8(53.3)$ & 1.0 \\
Aortic valve surgery & $5(33.3)$ & $3(20.0)$ & .409 \\
Aortic surgery & $2(13.3)$ & $1(6.7)$ & .543 \\
IABP timing & & & .456 \\
Intraoperative & $8(53.3)$ & $10(66.7)$ & \\
Postoperative & $7(46.7)$ & $5(33.3)$ & \\
Indications for IABP & & & .339 \\
Perioperative AMI & $3(20.0)$ & $2(13.3)$ & \\
Intraoperative myocardial & $7(46.7)$ & $4(26.7)$ & \\
$\quad$ stunning & & & \\
Difficult weaning from CPB & $5(33.3)$ & $9(60.0)$ & \\
\hline COPD was consder prsen & &
\end{tabular}

COPD was considered present if the patient had at least FEV1/FVC $<70 \%$ with FEV $1 \geq 50 \%$ and $<80 \%$ of predicted according to the GOLD classification (available at www.goldcopd.org). Kidney disease was considered present if the patient had at least an estimated glomerular filtration rate of 60 to $89 \mathrm{~mL} / \mathrm{min} / 1.73 \mathrm{~m}^{2}$ according to the KKDOQI classification (available at www.kdoqi.org). IABP, Intra-aortic balloon pump; $V$, weaning by progressive volume deflation; $R$, weaning by progressive ratio reduction; COPD, chronic obstructive pulmonary disease; euroSCORE, European System for Cardiac Operative Risk Evaluation; $L V E F$, left ventricular ejection fraction; $A C C$, aortic crossclamping; $C P B$, cardiopulmonary bypass; $C A B G$, coronary artery bypass grafting (distal anastomoses); $A M I$, acute myocardial infarction; FEVI/FVC, forced expiratory volume in 1 second/forced vital capacity; KKDOQI, Kidney Disease Outcomes Quality Initiative.

Perioperative glycemic control was achieved throughout the study protocol and during the entire ICU stay by continuous insulin infusion according to the Portland protocol. ${ }^{12}$ The institution's ethical committee/institutional review board approved the study protocol, and each patient provided informed consent.

\section{Statistical Analysis}

The enrollment of 30 patients (15 for each study arm) resulted in $84 \%$ power $(1-\beta$ error probability) that the study detected a treatment difference-for the periweaning CI-at a 2-sided .05 significance level, if the true difference between treatments was at least $20 \%$ of the mean of 1 treatment group in terms of the percentile of the mean of the other treatment group, as previously already reported. ${ }^{13}$ The partial $\eta^{2}$ of the model for 9-time CI measurement was 0.82 , with a calculated effect size f of 2.1. Accordingly, the clinical outcome data should be considered as descriptive only.

The pre- and perioperative data are summarized as the mean \pm standard deviation or median and 25th to 75th percentile, if continuous, and as counts and percentages, if categorical. The continuous variables were tested for normality with the Shapiro-Wilk test and compared between the 2 treatment groups with the Student $t$-test or the Mann Whitney $U$-test. Categorical variables were compared using the Fisher exact test.
Ordinal variables were also compared using Mann-Whitney $U$-test. Repeated measures analysis of variance with Bonferroni's correction for multiple measurements was used to compare serial data related to troponin I, lactate release, and the different hemodynamic indexes. Violations of sphericity were Greenhous-Geisser corrected if $\varepsilon<0.75$ or Huynh-Feldt corrected if $\varepsilon>0.75$. The reported $P$ values include group $P$, assessing the level of difference between groups; time $P$, assessing the change over time of the measured variables; group*time $P$, assessing the group-time interaction. Comparisons were considered significant at $P<.05$, unless dictated otherwise by Bonferroni's correction. Statistical analysis was performed using the SPSS program for Windows, version 15.0 (SPSS, Chicago, Ill).

\section{RESULTS}

The mean interval from IABP insertion for LCOS and the start of the weaning trial was comparable in the 2 groups (group V, $74.8 \pm 16.6$ hours vs group $\mathrm{R}, 72.0 \pm 15.8$ hours; $P=.641)$.

\section{Hemodynamic Response}

IABP-induced diastolic pressure augmentation was preserved in all the assisted beats during the 1:2 and 1:3 ratios of the weaning phase in group R. Diastolic augmentation was always preserved (although attenuated to an extent proportional to the weaning phase) at each step of the entire weaning trial in all patients in group $\mathrm{V}$.

Both groups started with a similar hemodynamic profile at the beginning of the weaning trial (T0, $P=.616$ for $\mathrm{CI}$, $P=.189$ for ISVR, $P=.393$ for CCE, and $P=.386$ for CVP; Figures 1 and 2). However, although both groups maintained a CI greater than $2.5 \mathrm{~L} / \mathrm{min} / \mathrm{m}^{2}$ during the entire study period, the $\mathrm{CI}$ values were overall greater in group $\mathrm{V}$ than in group $\mathrm{R}$ (between-group $P=.0001$; Figure $1, A$ ), because of the significantly better values in T5 to T8 (group*time $P=.0001$; Figure $1, A$ ). Similarly, greater vasodilation was achieved by volume deflation (ISVR between-group $P=.0001$; Figure $1, B$ ), particularly because of the significantly lower values for T2 to T8 (group*time $P=.0001$; Figure $1, B$ ).

The CCE declined at T1 in both groups (Figure 2). However, given the better CI and ISVR in patients undergoing volume deflation during the trial and subsequent ICU stay, hemodynamic monitoring also revealed significantly better CCE (between-group $P=.0001$; Figure 2, $A$ ), mainly as a result of the better values for T3 to T8 (group*time, $P=.0001$; Figure 2, A). Finally, the CVP was lower in group $\mathrm{V}$ (between-group $P=.0001$; Figure $2, B$ ) for T4 to T8 (group*time $P=.0001$; Figure $2, B$ ).

\section{Biochemical Results}

The troponin I and lactate levels were similar at the beginning of the trial $(P=.242$ and $P=.199$, respectively, at T0; Table 2), and no differences were found in terms of troponin (between-group $P=.715$ ) at any point (group*time $P=.689$; Table 2). However, the serum lactate 

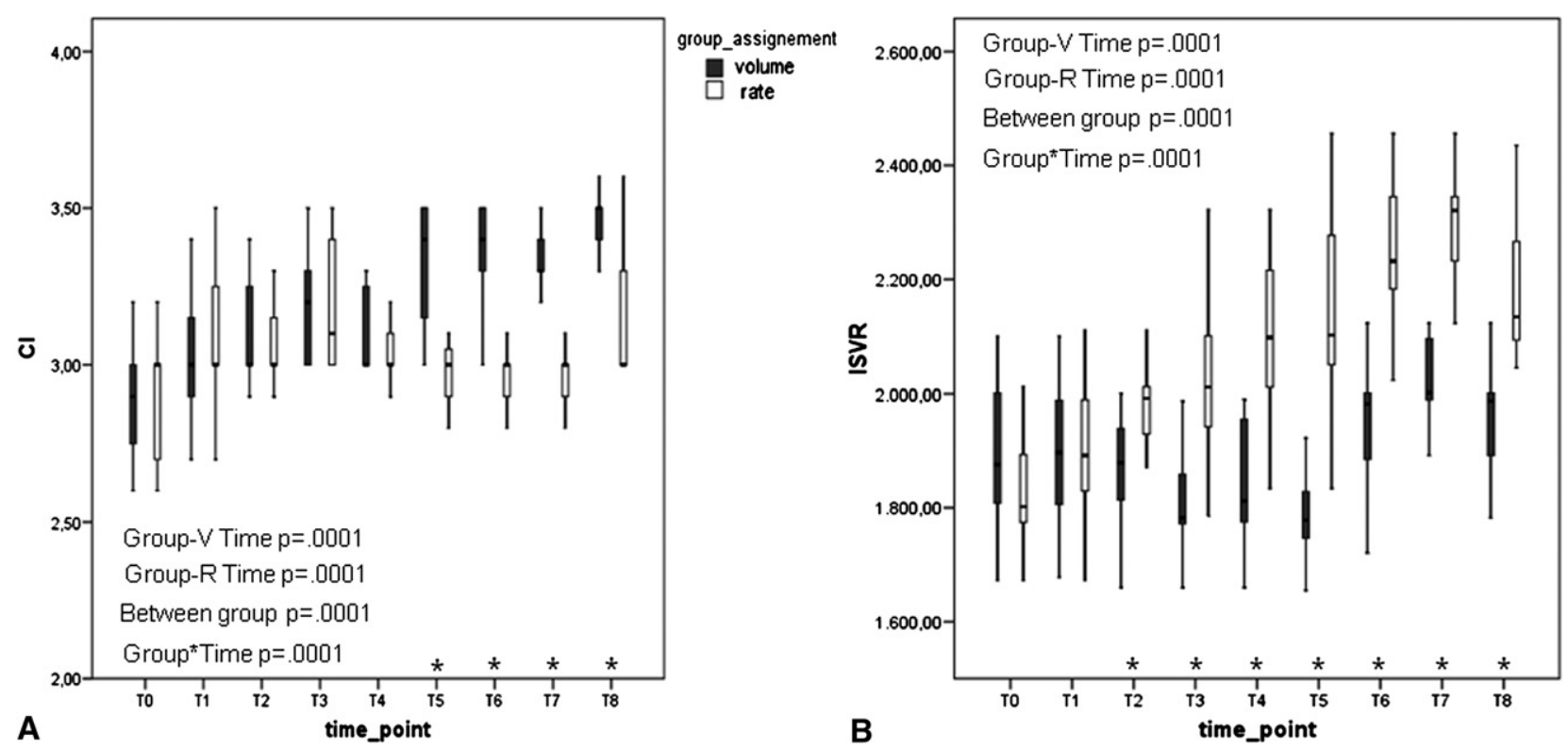

FIGURE 1. A, Cardiac index (CI) and (B) indexed systemic vascular resistance (ISVR). T0 to T8 as reported in the text. Values are presented as the mean \pm standard deviation with $95 \%$ confidence intervals. Time-point asterisks indicate significant values at single intervals (univariate analysis). $V$, Weaning by progressive volume deflation; $R$, weaning by progressive ratio reduction.

level was lower in group $\mathrm{V}$ (between-group $P=.002$; Table 2 ), owing to the lower values for T5 to T8 (group*time $P=.001$; Table 2).

\section{Clinical Outcomes}

One patient in group R (3.3\% of the entire study population) showed progressive hemodynamic impairment during the weaning trial at $\mathrm{T} 4\left(\mathrm{CI}, 2.1 \mathrm{~L} / \mathrm{min} / \mathrm{m}^{2}\right.$, ISVR, 2212 dyne/ $\mathrm{s} / \mathrm{cm}^{-5} / \mathrm{m}^{2}, \mathrm{CCE},-0.21, \mathrm{CVP}, 16 \mathrm{~mm} \mathrm{Hg}$ ). This patient required full IABP assistance until the sixth hour from the start of weaning. At the sixth hour, a step-down to a 1:2 assist ratio to the eighth hour and to $1: 3$ for the next hour was successful (weaning failure in group R, 1/15 [6.7\%] and in group $\mathrm{V}, 0 / 15 ; P=1.0$ ). Eventually, all patients were successfully weaned from IABP, with no need for IABP reinstitution after its withdrawal. No differences were found
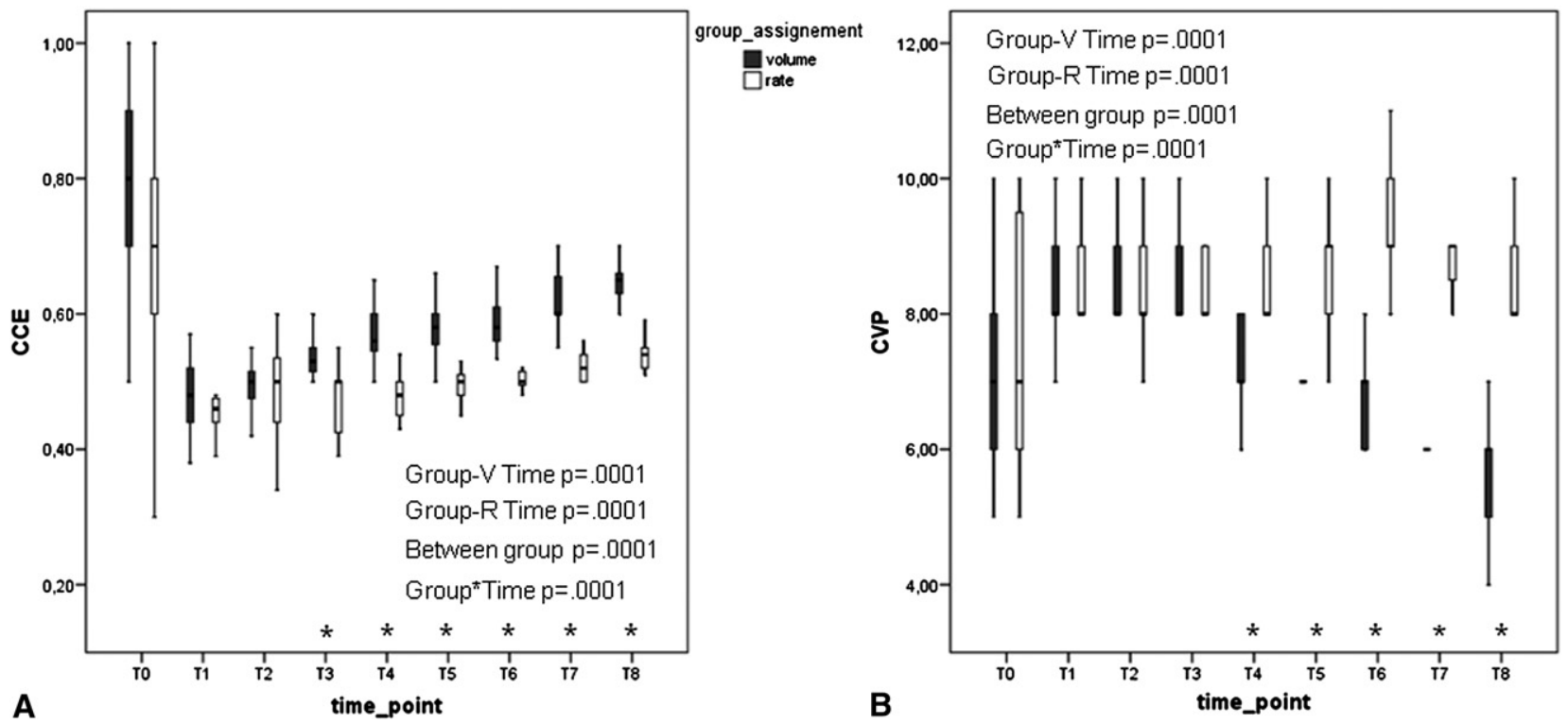

FIGURE 2. A, Cardiac cycle efficiency $(C C E)$ and (B) central venous pressure $(C V P)$. T0 to T8 as reported in the text. Values are presented as the mean \pm standard deviation with $95 \%$ confidence intervals. Time-point asterisks indicate significant values at single intervals (univariate analysis). $V$, Weaning by progressive volume deflation; $R$, weaning by progressive ratio reduction. 
TABLE 2. Biochemical results

\begin{tabular}{|c|c|c|c|c|c|c|c|c|c|c|c|}
\hline & T0 & T1 & T2 & T3 & T4 & T5 & T6 & T7 & T8 & Time- $P$ value & $\begin{array}{c}\text { Between-group } \\
P \text { value }\end{array}$ \\
\hline \multicolumn{12}{|c|}{ Troponin I $(\mu \mathrm{g} / \mathrm{L})$} \\
\hline Group V & $5.8 \pm 2.1$ & $5.0 \pm 0.9$ & $5.1 \pm 0.9$ & $4.9 \pm 0.9$ & $4.7 \pm 1.0$ & $4.4 \pm 1.0$ & $4.3 \pm 0.9$ & $3.8 \pm 1.0$ & $3.3 \pm 0.9$ & .001 & .715 \\
\hline Group R & $6.5 \pm 1.1$ & $5.1 \pm 1.3$ & $4.9 \pm 1.4$ & $4.8 \pm 1.2$ & $4.6 \pm 1.2$ & $4.4 \pm 1.0$ & $4.2 \pm 1.0$ & $4.1 \pm 1.0$ & $3.9 \pm 0.9$ & .002 & \\
\hline$P$ value* & .242 & .897 & .801 & .726 & .794 & .986 & .940 & .396 & .070 & $\begin{array}{c}\text { Group*time } \\
P \text { value }\end{array}$ & .689 \\
\hline \multicolumn{12}{|c|}{ Lactate $(\mathrm{mmol} / \mathrm{L})$} \\
\hline Group V & $2.3 \pm 0.5$ & $2.2 \pm 0.7$ & $2.2 \pm 0.7$ & $2.1 \pm 0.7$ & $2.0 \pm 0.7$ & $1.9 \pm 0.7$ & $1.5 \pm 0.7$ & $1.2 \pm 0.5$ & $1.2 \pm 0.7$ & .0001 & .002 \\
\hline Group R & $2.1 \pm 0.5$ & $2.3 \pm 1.3$ & $2.3 \pm 1.2$ & $2.2 \pm 1.2$ & $2.6 \pm 0.8$ & $2.8 \pm 0.7$ & $2.1 \pm 0.6$ & $1.8 \pm 0.6$ & $1.8 \pm 0.6$ & .007 & \\
\hline$P$ value* & .199 & .725 & .695 & .714 & .074 & .002 & .019 & .010 & .027 & $\begin{array}{c}\text { Group*time } \\
P \text { value }\end{array}$ & .001 \\
\hline
\end{tabular}

Data are presented as mean \pm standard deviation. T0 to $\mathrm{T} 8$ as reported in the text. $V$, Weaning by progressive volume deflation; $R$, weaning by progressive ratio reduction. $*$ Single points (univariate analysis)

between the 2 groups in the amount of inotropic support during the weaning trial (group $\mathrm{V}$, low-dose, 2/15 [13.3\%]; medium-dose, 10/15 [66.7\%]; high-dose, 3/15 [20.0\%] vs group R, low-dose, $3 / 15[20.0 \%]$, mediumdose, $8 / 15$ [53.3\%], high-dose, $4 / 15$ [28.7\%]; $P=.753$ ). One patient in group $\mathrm{V}(6.7 \%)$ and 3 patients in group $\mathrm{R}(20.0 \% ; P=.598)$, however, required postwithdrawal inotropic augmentation to achieve a satisfactory hemodynamic profile.

In group R, 2 (13.3\%) of 15 patients died in hospital, 1 of pneumonia and 1 of stroke. No patient in group $\mathrm{V}$ died $(P=.483)$. No postwithdrawal AMI occurred. Postwithdrawal paroxysmal/persistent atrial fibrillation developed in 6 patients in group $\mathrm{R}(40.0 \%)$ and 5 patients in group $\mathrm{V}(33.3 \% ; P=1.0)$.

Patients randomized to progressive volume deflation had a quicker discharge from the ICU after IABP withdrawal (18.6 \pm 0.6 vs $20.8 \pm 1.4$ hours in group $\mathrm{R} ; P=.0001)$. However, no differences were detected in overall ICU stay (group V, $7.8 \pm 1.9$ days vs group R, $9.0 \pm 1.7$ days; $P=.097$ ) or hospitalization (group $\mathrm{V}, 17.4 \pm 2.7$ days vs group R, $18.3 \pm 1.9$ days; $P=.359$ ). No thrombotic, embolic, hemorrhagic, septic, or vascular complications were reported.

\section{DISCUSSION}

The IABP is the most commonly used left ventricular assist device to manage heart failure, and its use has been foreseen to increase owing to the referral of progressively sicker patients to the ICU. ${ }^{3,6}$ Despite its worldwide use, however, to the best of our knowledge, no studies have yet evaluated which method would be the best to wean a patient from IABP therapy. This issue is of utmost importance given the profound effect of LCOS on patient survival, quality of life, and use of public health resources. ${ }^{7,14}$

Furthermore, ventricular unloading saves viable myocardium and plays a crucial role in remodeling. ${ }^{15}$ Accordingly, the weaning phase from IABP therapy should be seriously planned and interpreted as the last step of the myocardial protective strategy. ${ }^{14}$ Our study first assessed the hemodynamic response to 2 different weaning methods. We found a definitely better hemodynamic profile in patients weaned by progressive volume deflation compared with those weaned by a reduction of the assist rate, as demonstrated by the greater degree of vasodilation, the better CI, the lower oxygen consumption of the myocardium (indirectly derived from the CCE), and the lower production of peripheral lactate. A recent survey from Australia showed eclectic approaches to IABP weaning in different hospitals (eg, isolated rate reduction, isolated volume deflation, and different combinations of both). ${ }^{16}$ Isolated rate reduction was found to be the best method to lessen the incidence of IABP reinstitution and to prevent the institution/augmentation of inotropic support. ${ }^{16}$ However, that study was not targeted to investigate the best weaning method. ${ }^{16}$ Furthermore, the absence of standard weaning protocols and hemodynamic monitoring, the excessive case mix in terms of indications for IABP (pre-, intra-, and postoperative), and the extreme variability in the skills and volume of IABP practice in the different centers significantly affected the results of that study. ${ }^{16}$ Finally, these investigators acknowledged that their results were the consequence of a volume-outcome relationship rather than dependent on a beneficial hemodynamic effect, with high-volume centers (more familiar with IABP) generally using the rate-reduction weaning method. ${ }^{16}$ The strengths of our study were the prospective randomized design and that we studied only the intra- and postoperative IABP for LCOS (preoperative IABP use was excluded because of the completely different hemodynamic profile of these patients). Also, we only included IABP use after cardiac surgery with cardiopulmonary bypass. Cardiologic or other clinical scenarios were also excluded because of the completely different hemodynamic settings. Finally, a strength of our study was that it was targeted to different hemodynamic and biochemical parameters to comprehensively evaluate myocardial performance, cardiac work, and systemic perfusion and metabolism. 
Although we found a quicker discharge from the ICU after IABP withdrawal by volume deflation, the overall ICU stay and hospital stay proved comparable in the 2 groups. This could have been a consequence of the limited number of patients enrolled (related to the rare occurrence of LCOS after surgery in current practice). However, although the differences in ICU stay and overall hospitalization did not reach statistical significance, group $\mathrm{V}$ patients spent an average of 1 day less in either the ICU or the hospital compared with group R. Both the value of these differences and their effect on the use of economic resources deserve future studies.

It is well-known that LCOS has a major influence on microflow and vascular density, because the smallest vessels are the principle ones responsible for the altered perfusion. ${ }^{17}$ Oxygen use is consequently limited by inadequate transport, leading to increased blood lactate production. ${ }^{17}$ It has been proved that the greater the lactate levels, the lower the flow in the microcirculation. ${ }^{18}$ Furthermore, previous studies have demonstrated that the microflow changes induced by cardiogenic shock can be pharmacologically reversed, ${ }^{17}$ suggesting that therapeutic interventions aimed at opening the microcirculation could be beneficial. It has been shown that IABP therapy is able to completely reverse the microflow changes induced by shock, by way of an endothelial-mediated mechanism, and leading to acute microflow improvement. ${ }^{18}$ Thus, our data suggest that progressive deflation of the balloon volume better preserves the blood flow in the microcirculation, promoting more efficient oxygen delivery. Munsterman and colleauges ${ }^{19}$ in a recent experimental study of human sublingual microcirculation showed that microcirculatory flow paradoxically increases after stopping the IABP, suggesting an impairment effect of the IABP on microcirculation. It should be recognized, however, that the study design (1:1 IABP followed by cyclic cessation) used by Munsterman and colleagues $^{19}$ mimics our weaning method using rate reduction, suggesting that our findings in terms of lactate leakage could be interpreted as resulting from a hampered systemic microcirculation achieved using the 1:2 assist mode. However, it should be acknowledged that the study by Munsterman and colleagues ${ }^{19}$ was flawed by the absence of any hemodynamic monitoring, the extreme variability in the indications for IABP, and the study design — with IABP abrupt cessation-not mimicking at all the routine practice of IABP therapy. ${ }^{19}$ Finally, the clinical value of sublingual microcirculation and its correspondence with that of other organs has been questioned by some studies, showing how sublingual microflow changes do not directly correlate with the microflow changes of gut and brain, which can often be opposite those of the sublingual circulation. ${ }^{20,21}$ Thus, more studies on this topic are warranted.

We were able to demonstrate that IABP weaning by volume deflation reduced peripheral lactate leakage. Jung and coworkers ${ }^{18}$ proved that not, simplistically, the CI, but the cardiac perfusion index (related to both cardiac performance and peripheral perfusion) correlated with circulating lactate levels. This demonstrated that overall lactate production during LCOS is not just of cardiac origin, but rather the combined result of cardiac dysfunction and altered peripheral oxygen delivery. ${ }^{18}$ Similarly, our findings have confirmed that compared with rate reduction, the volumedeflation strategy translates into greater peripheral oxygen delivery and better myocardial metabolism, indicating the latter method is potentially more physiologic. The better myocardial metabolism might translate into a greater $\mathrm{CI}$ and CCE, indicating better coupling between cardiac function and energy expenditure. ${ }^{9}$ Similar to the cardiac perfusion index of the study by Jung and colleagues, ${ }^{18}$ the lower myocardial oxygen consumption (CCE) and the greater peripheral perfusion (ISVR) translated into lower serum lactate leakage in group $\mathrm{V}$.

It is also noteworthy that an evident decline of the CCE was already present at $\mathrm{T} 1 \mathrm{in}$ both groups, although this was subsequently attenuated by the volume-deflation strategy. An elegant in vivo study has already demonstrated that IABP reduced myocardial oxygen demand and improved distal perfusion owing to the augmentation of aortic elastance. ${ }^{22}$ Similarly, the IABP induces an augmentation of stroke work, a reduction of myocardial oxygen demand, decreased systemic vascular resistance, and aortic impedance. ${ }^{23}$ Bia and colleagues ${ }^{24}$ and Cabrera-Fischer and colleagues ${ }^{25}$ showed that the IABP has a direct vasodilative effect on the smooth muscle cells. A direct endotheliumdependent vasoactive effect has also been shown on small and medium arterioles ${ }^{26}$ and at a microvascular level. ${ }^{18}$ According to these peripheral effects of IABP, the lower ISVR in group $\mathrm{V}$ of our study suggested maintenance during the weaning period of the vasodilative effect of the balloon, with more pronounced vascular effects with the volume-deflation weaning method. Finally, a recent human study evaluated the balloon compression flow wave (during inflation) and its expansion flow wave (during deflation) with a $1: 1$ or $1: 2$ assist ratio and correlated them to diastolic aortic pressure augmentation and reduction. ${ }^{27}$ The investigators found that the diastolic aortic pressure augmentation was greater during the 1:1 ratio and enddiastolic aortic pressure reduction decreased during the 1:1 ratio but was unchanged during the $1: 2$ ratio. ${ }^{27}$ The energy of the compression wave correlated positively with the diastolic pressure augmentation and the energy of the expansion wave correlated negatively with the enddiastolic aortic pressure reduction only with the 1:1 assist ratio. $^{27}$ In contrast, these relationships were unchanged during the 1:2 ratio. $^{27}$ All these data demonstrated that 1:2 IABP therapy does not result in any hemodynamic benefit. ${ }^{27}$ The results of our study similarly suggested that switching an IABP to the 1:2 ratio simply corresponded 
to the loss of any ventricular assistance, translating to the abrupt cessation of IABP therapy.

From the results of our study, it could be speculated that the rate-reduction weaning method might be hazardous early after LCOS. However, the progressive volume deflation-with its better preserved hemodynamic profile-appears to reduce the IABP assist properties more gradually, aiding the healing ventricle to undergo a more physiologic weaning trial.

\section{Study Limitations}

The main limitation of the present study was the small sample size (resulting from the preliminary nature of the trial), significantly limiting the clinical significance of the reported differences in hemodynamic and biochemical outcome variables. It also reflects the current low incidence of severe postcardiotomy LCOS requiring IABP therapy. Finally, our study was a single-center design, although that guaranteed the uniformity of the perioperative management of the patient population and strict adherence to the planned protocol.

The present study lacked pure cardiologic indications for IABP (eg, AMI in the setting of an ICU) outside the cardiac surgery scenarios. Therefore, our patients could presumably have benefited from the surgical relief of myocardial ischemia and/or of hemodynamic compromise caused by the underlying valve disease. Thus, the IABP weaning in our series might have incorporated some physiologic improvements unrelated to the sole IABP. Although our data cannot be directly extrapolated to different clinical scenarios, the results represent the first comparison with hemodynamic monitoring of 2 different weaning modalities in the postoperative phase of cardiac surgical practice.

The similar clinical outcomes obtained with the 2 techniques might suggest that both IABP weaning methods can be considered effective if a patient has been adequately supported and allowed to recover. However, the results of the present study highlight how different the hemodynamic and biochemical responses to the 2 weaning methods can be (despite an apparently comparable and uncomplicated clinical outcome). The better hemodynamic indexes and lower peripheral lactate leakage after progressive volume deflation might suggest a new thinking in the approach to the IABP weaning phase. More studies with a larger number of patients are necessary to investigate the actual clinical effect of these 2 different strategies.

Another limitation was related to the absence of objective data precisely quantifying the entity of progressive volume deflation at each cycle. The IABP console has been validated for progressive volume deflation in clinical practice, and internal safety systems are able to continuously monitor the inner volume of the entire circuit and to quantify the volume of inflation and deflation for each cardiac cycle. However, experimental models with peripheral flow meters or magnetic resonance imaging studies with computational models measuring the degree of displaced blood volume for each IABP cycle are probably needed to better quantify the volume deflation weaning method.

A final limitation was the absence of standardized IABP weaning methods in the current data to which refer for our study design and method. Therefore, ethical issues led us to use the only 2 available IABP weaning methods, to adhere to our traditional institutional policies, and to prospectively randomize patients to 1 of these 2 methods. From a different perspective, these limitations could be considered as strengths of our study. This is because it is first prospective randomized trial to evaluate the best IABP weaning method using a comprehensive biochemical, hemodynamic, and clinical outcome analysis for a select cohort of patients with postcardiotomy LCOS. This also excluded patients undergoing prophylactic preoperative IABP. The results of the present study deserve future investigations to better define the role of different IABP weaning methods on subtle microvascular flow changes, different scenarios (eg, prophylactic IABP), different organ function, and so on.

\section{CONCLUSIONS}

The preliminary results of our pilot study seem to suggest that IABP weaning using progressive volume deflation should be preferred to the traditional rate reduction because of the better hemodynamic and metabolic profiles, as shown by the better CI, greater degree of vasodilation, lower oxygen consumption of the myocardium (indirectly derived from the CCE), and lower production of peripheral lactate. Despite the apparently similar and satisfactory clinical outcomes, the hemodynamic and biochemical responses to the 2 weaning protocols proved to be completely different. The volume-deflation method was a gradual and slower approach to IABP weaning, and the rate-reduction method resulted in abrupt IABP cessation, with the potential to harm a recently healed ventricle.

\section{References}

1. Bolooki H, Williams W, Thurer RJ, Vargas A, Kaiser GA, Mack F, et al. Clinical and hemodynamic criteria for the use of the intra-aortic balloon pump in patients requiring cardiac surgery. J Thorac Cardiovasc Surg. 1976;72:756-8.

2. Kawaguchi O, Pae WE, Daily BB, Pierce WS. Ventriculoarterial coupling with intra-aortic balloon pump in acute ischemic heart failure. J Thorac Cardiovasc Surg. 1999;117:164-71

3. Ferguson JJ III, Cohen M, Freedman RJ, Stone GW, Miller MF, Joseph DL, et al. The current practice of intra-aortic balloon counterpulsation: results from the Benchmark Registry. J Am Coll Cardiol. 2001;38:1456-62.

4. Baskett RJ, Ghali WA, Maitland A, Hirsch GM. The intraaortic balloon pump in cardiac surgery. Ann Thorac Surg. 2002;74:1276-87.

5. Onorati F, Bilotta M, Pezzo F, Impiombato B, Caroleo S, Santangelo E, et al. Transbrachial insertion of a $7.5 \mathrm{Fr}$ intra-aortic balloon pump in a severely atherosclerotic patient. Crit Care Med. 2006;34:2231-3.

6. National Center for Health Statistics. National Hospital Discharge Survey: 2009 annual summary with detailed diagnosis and procedure data. Available at: http://www.cdc.gov/nchs/data/nhds/10Detaileddiagnosesprocedures/2009det10_ allistedprocedures.pdf. Accessed April 13, 2012.

7. Onorati F, De Feo M, Mastroroberto P, Cristodoro L, Pezzo F, Renzulli A, et al. Determinants and prognosis of myocardial damage after coronary artery bypass grafting. Ann Thorac Surg. 2005;79:837-45. 
8. Zangrillo A, Maj G, Monaco F, Scandroglio AM, Nuzzi M, Plumari V, et al. Cardiac index validation using the pressure recording analytic method in unstable patients. J Cardiothorac Vasc Anesth. 2010;24:265-9.

9. Scolletta S, Ranaldi G, Carlucci F, Franchi F, Romano SM, Biagioli B. Relationship between N-terminal pro-B-type natriuretico peptide (NT-proBNP) and cardiac cycle efficiency in cardiac surgery. Biomed Pharmacother. 2010;64:511-5.

10. Thygesen K, Alpert JS, White HD, Jaffe AS, Apple FS, Galvani M, et al, on behalf of the Joint ESC/ACCF/AHA/WHF Task force for the Redefinition of Myocardial Infarction. Universal definition of myocardial infarction. $J$ Am Coll Cardiol. 2007;50:2173-95.

11. Onorati F, Curcio A, Santarpino G, Torella D, Mastroroberto P, Tucci L, Indolfi $\mathrm{C}$, Renzulli A. Routine ganglionic plexi ablation during Maze procedure improves hospital and early follow-up results of mitral surgery. J Thorac Cardiovasc Surg. 2008;136:408-18.

12. Zerr KJ, Furnary PA, Grunkemeier GL, Bookin S, Kanhere V, Starr A. Glucose control lowers the risk of wound infection in diabetics after open heart operations. Ann Thorac Surg. 1997;63:356-61.

13. Stefanadis C, Dernellis J, Tsiamis E, Stratos C, Kallikazaros I, Toutouzas P. Aortic function in patients during intra-aortic balloon pumping determined by the pressure-diameter relation. J Thorac Cardiovasc Surg. 1998;116:1052-9.

14. Mentzer RM, Jahania MS, Lasley R. Myocardial protection. In: Cohn LH, ed. Cardiac Surgery in the Adult. New-York: McGraw-Hill; 2008. p. 443-64.

15. Deschamps AM, Apple KA, Leonardi AH, McLean JE, Yarbrough WM, Stroud RE, et al. Myocardial interstitial matrix metalloproteinase activity is altered by mechanical changes in LV load: interaction with angiotensin type 1 receptor. Circ Res. 2005;96:1110-8.

16. Lewis PA, Mullany DV, Courtney M, Coyer F. Australasian trends in intra-aortic balloon counterpulsation weaning: results of a postal survey. Crit Care Resusc. 2006;8:361-7.

17. De Backer D, Creteur J, Dubois MJ, Sakr Y, Vincent JL. Microvascular alterations in patients with acute severe heart failure and cardiogenic shock. Am Heart J. 2004;147:91-9.
18. Jung C, Rodiger C, Fritzenwanger M, Schumm J, Lauten A, Figulla HR, et al. Acute microflow changes after stop and start of intraaortic balloon pump in cardiogenic shock. Clin Res Cardiol. 2009;98:469-75.

19. Munsterman LD, Elbers PW, Ozdemir A, van Dongen EP, van Iterson M, Ince C Withdrawing intra-aortic balloon pump support paradoxically improves microvascular flow. Crit Care. 2010;14:R161.

20. Verdant CL, De Backer D, Bruhn A, Clausi CM, Su F, Wang Z, et al. Evaluation of sublingual and gut mucosal microcirculation in sepsis: a quantitative analysis. Crit Care Med. 2009;37:2875-81.

21. Wan Z, Sun S, Ristagno G, Weil MH, Tang W. The cerebral microcirculation is protected during experimental hemorrhagic shock. Crit Care Med. 2010;38: 928-32.

22. Stefanadis C, Dernellis J, Tsiamis E, Stratos C, Kallikazaros I, Toutouzas P Aortic function in patients during intra-aortic balloon pumping determined by the pressure-diameter relation. J Thorac Cardiovasc Surg. 1998;116: 1052-9.

23. Kim SY, Euler DE, Jacobs WR, Montoya A, Sullivan HJ, Lonchyna VA, et al Arterial impedance in patients during intraaortic balloon counterpulsation. Ann Thorac Surg. 1996;61:888-94.

24. Bia D, Zócalo Y, Armentano R, Forteza E, Cabrera-Fischer EI. Increased reversal and oscillatory shear stress cause smooth muscle contraction-dependent changes in sheep aortic dynamics: role in aortic balloon pump circulatory support. Acto Physiol (Oxf). 2008;192:487-503.

25. Cabrera-Fischer EI, Bia D, Zócalo Y, Armentano RL. Smooth muscle-dependent changes in aortic wall dynamics during intra-aortic counterpulsation in an animal model of acute heart failure. Int J Artif Organs. 2009;32:354-61.

26. Toyota E, Goto M, Nakamoto H, Ebata J, Tachibana H, Hiramatsu O, et al. Endothelium-derived nitric oxide enhances the effect of intraaortic balloon pumping on diastolic coronary flow. Ann Thorac Surg. 1999;67:1254-61.

27. Kolyva C, Pantalos GM, Giridharan GA, Pepper JR, Khir AW. Discerning aortic waves during intra-aortic balloon pumping and their relation to benefits of counterpulsation in humans. J Appl Physiol. 2009;107:1497-503. 